\title{
A Case of Solid Variant of Adenoid Cystic Carcinoma from Trachea: A Case Report and Review of Literature
}

This article was published in the following Dove Press journal: OncoTargets and Therapy

\section{Feng Gao \\ Lijuan Zang \\ Jin $\mathrm{He}$ \\ Weiqing $\mathrm{Xu}$}

Department of Pathology, Shanghai General Hospital,Shanghai Jiao Tong University School of Medicine, Shanghai, 200080, People's Republic of China
Correspondence: Weiqing $\mathrm{Xu}$ Department of Pathology, Shanghai General Hospital, Shanghai Jiao Tong University School of Medicine, No. 85/86 Wujin Road, Hongkou District, Shanghai, 200080, People's Republic of China

Tel +86-2I-I3661888020

Email xuweiqimg@I63.com
Introduction: Primary tracheal adenoid cystic carcinoma (ACC) is a rare and heterogeneous group of neoplasms arising from the respiratory tract. The solid variant of ACC is a histologically distinct subtype with an unfavorable clinical course. We report on a case of tracheal ACC with immunohistochemical and molecular analysis together with a review of the literature.

Case Report: We observed a case in which a 31-year-old male presented with a neoplasm bulging into the lumen and caused symptoms of tracheal obstruction and even hemoptysis. Cytological smears of an endobronchial fine needle aspiration revealed aggregates of basaloid cells with small to medium size, scant cytoplasm, and evenly hyperchromatic nuclei. Histologically, the tumor is characterized by a predominant compact sheet-like and nested pattern of rounded basaloid cells. Immunohistochemically, the tumor was diffusely positive for CK and CD117. CK7 and CK5/6 were focally positive in the genuine glandular structures. P63 was completely negative in the majority of neoplastic cells. Fluorescence in situ hybridization analysis revealed MYB gene rearrangement.

Conclusion: The solid variant of ACC from trachea is rare and hence poses diagnostic difficulty. Computed tomography (CT) scan and bronchoscopy help assess the extent of the disease. Histological features combined with immunophenotypic and molecular analysis aid in distinguishing this uncommon type from other round cell neoplasms. The accurate diagnosis can help expedite treatment of this highly aggressive tumor.

Keywords: tracheal neoplasm, adenoid cystic carcinoma, clinical pathology, immunohistochemistry, MYB

\section{Background}

Adenoid cystic carcinoma (ACC) is a rare, distinctive salivary gland malignant neoplasm infrequently arising as a primary tumor in the lung. ${ }^{1}$ ACC often arises in the trachea and main bronchus. Morphologically, this subset of primary pulmonary carcinoma is histologically indistinguishable from ACC arising from other sites. ACC can have three architectural growth patterns: cribriform, tubular, and solid, with a cribriform pattern being the most common type. The solid variant of ACC is a histologically distinct subtype that has been associated with an unfavorable clinical course. ${ }^{2,3}$ We report on a case of a solid tracheal variant of ACC that has a striking basaloid appearance. We performed a clinicopathological, immunophenotypic, and molecular analysis to delineate the characteristic features of this rare subtype. We highlight the need to recognize ACC in its solid form, discuss the differential diagnosis, and emphasize the need to establish a correct diagnosis for appropriate clinical management. 


\section{Case Presentation}

A 31-year-old Chinese man presented to our hospital with the chief complaints of cough, expectorations, and hemoptysis for two weeks without apparent cause. He denied other symptoms, including dyspnea, chest pain, fever, and recent weight loss. The patient's chest computed tomography (CT) demonstrated multiple lymph nodes enlargement, tracheal and esophageal compression, and multiple lung nodules in the horizontal mediastinal window section. Positron emission tomography (PET) showed lower tracheal nodules, multiple infiltrations in the lower tracheal and left main bronchial wall, multiple mediastinal lymph nodes enlargement, and multiple bone lesions throughout the body, which suggested the possibility of tracheal carcinoma with multiple metastases. The patient underwent a fiberoptic bronchoscopy. A neoplasm was detected on the lower part of the total tracheal membrane, left side wall, and anterior wall, arising at $3 \mathrm{~cm}$ distal to the upper segment of the left main bronchus from the protrusion of trachea (Figure 1). An endobronchial fine needle aspiration (FNA) was performed on the mass under bronchoscopic guidance, and FNA smears were made. The cytologic smears were highly cellular and contained 3-dimensional clusters of neoplastic basaloid cells, which are rarely associated with the hyaline basement membrane material. The individual tumor cells were homogeneous in size. The neoplasm consisted of aggregates of basaloid cells with small to medium size, scant cytoplasm, and evenly hyperchromatic nuclei (Figure 2).

Bronchofiberscope biopsy tissue was observed under the microscope (Figure 3). The surface of the tumor tissue was covered by pseudostratified columnar respiratory-type epithelium. The tumor exhibited a predominantly solid (>90\%) architecture comprised of basaloid-appearing cells. The solid nests were distributed under the mucosa and had microscopically invasive borders. The solid area, showing "geographic," irregular, or "garland-like" islands, could also be seen in the local shape of a sieve or trabecular structure. The intervening stroma was typically fibromyxoid or hyalinized. The neoplastic cells had a predominantly basaloid appearance characterized by small to medium cells. The surrounding cells were arranged in a palisade. The tumor

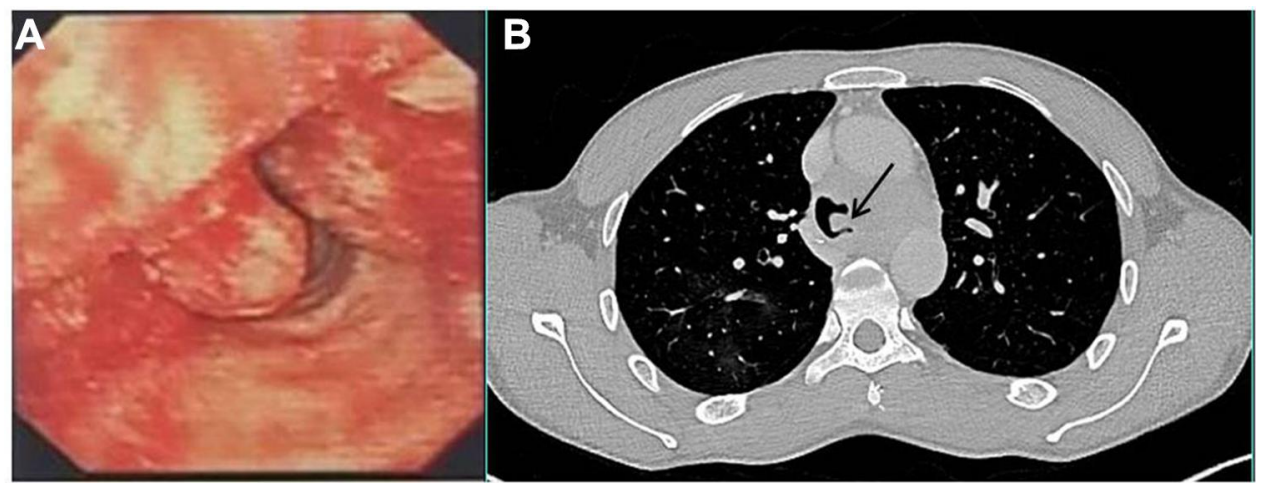

Figure I (A) Fiberoptic bronchoscopy examination demonstrating endoluminal growth, with submucosal infiltration with prominent mucosal vascularity causing occlusion of the bronchus intermedius. (B) CT scan of the thorax (mediastinal window section) demonstrating a subcarinal mass (black arrow) with soft tissue opacity in the left main bronchus.

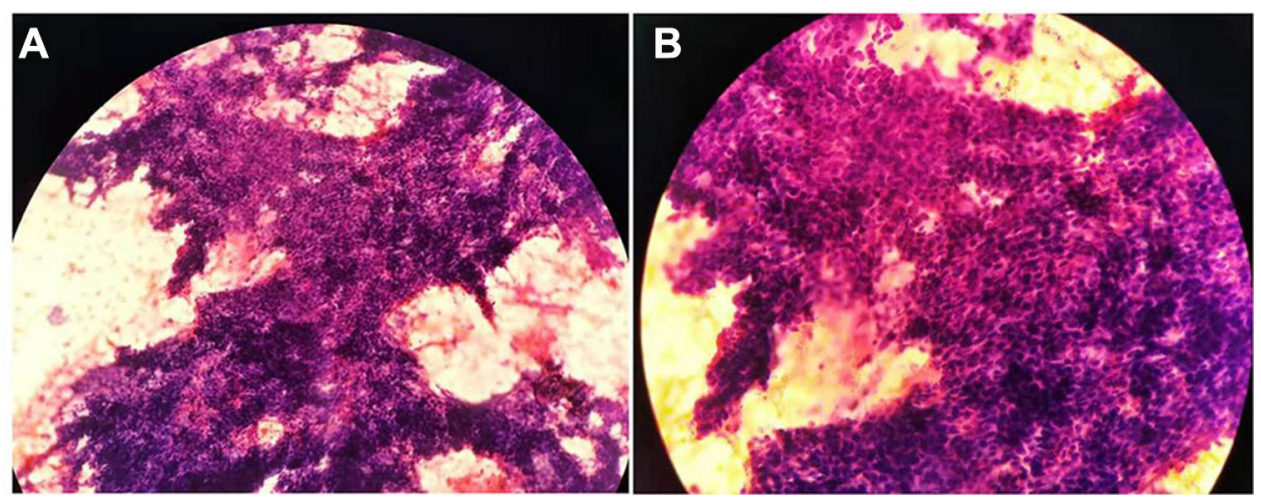

Figure 2 Cytological findings in the tracheal tumor. (A) The cytologic smears were highly cellular and contained 3-dimensional clusters of neoplastic basaloid cells. (B) Cohesive epithelial nest composed of relatively uniform epithelial cells with scant cytoplasm and hyperchromatic nuclei. 

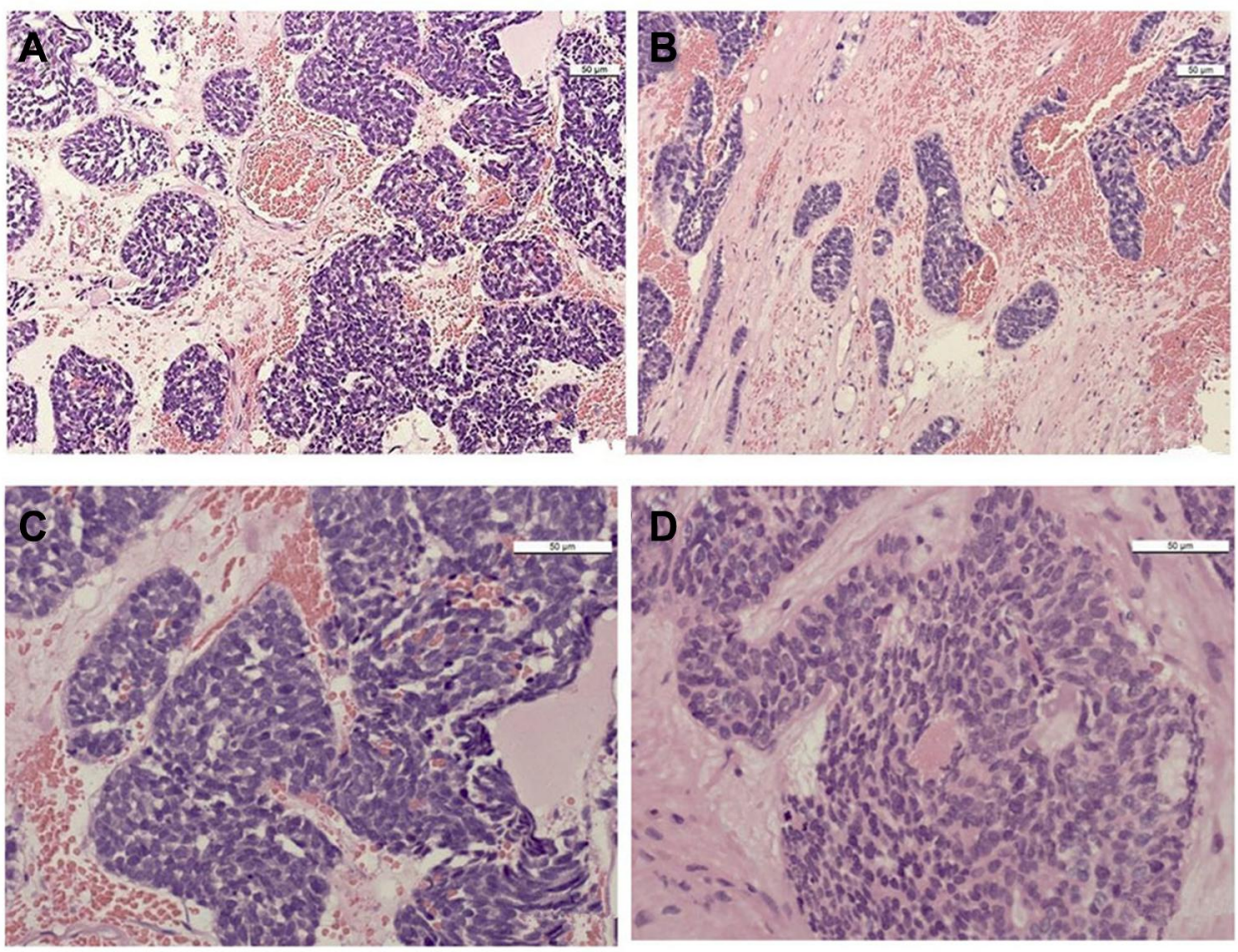

Figure 3 Histological findings of the tumor. (A) The tumor cells were in irregular nests and distributed in a fibrous stroma. (B) Some of the tumor cells grew in the shape of a beam. (C) The tumor cells were solid nests and showed basaloid features. The cells were round or ovoid with scant cytoplasm and hyperchromatic nuclei. (D) In some solid nests, the lumen-like structures were not obvious.

cells showed scant, mildly eosinophilic cytoplasm with a round to oval hyperchromatic nuclei and inconspicuous nucleoli. Nuclear atypia was moderate to marked. Mitotic activity was observed. Intercalated ducts within solid nests were poorly formed. Occasionally pseudoglandular structures were also seen with cribriform or glands with various sizes. These spaces were filled with homogenous, eosinophilic material. Focal necrosis was present in the nests of tumor cells.

The tumor tissue sections were immunostained with primary antibodies against broad-spectrum cytokeratin (CK), CK7, p63, p40, CD117 (c-kit), CK5/6, Calponin, thyroid transcription factor-1 (TTF-1), Napsin A, LCA, FLI-1, S-100, Synaptophysin (Syn), Chromatin A (chgA) and CD56. All antibodies were purchased from Gene Company. After incubation with a primary antibody, the detection of antibodies was accomplished using the Streptavidin-peroxidase method. Immunohistochemically, the tumor was diffusely positive for CK and CD117. CK 7 and CK5/6 were focally positive in the genuine glandular structures. P63, specific for myoepithelial cells, was completely negative in the majority of the neoplastic cells. Focal weak staining was seen in a small area. The tumor was negative for $\mathrm{p} 40$, Calponin, TTF-1, Napsin A, LCA, FLI-1, S-100, Syn, chgA, and CD56. The percentage of cells that stained positively for ki-67 was about $20-30 \%$ (Figure 4, Table 1).

Fluorescence in situ hybridization (FISH) was performed on 5-um-thick paraffin sections (Figure 5). A dual-color break-apart interphase FISH assay was performed using centromeric (BAC clone RP11-349 $\mathrm{J} 5$, red) and telomeric (BAC clone RP11-641019, green) probes to detect MYB rearrangement. MYB break-apart assay: Nuclei with a positive MYB breakapart signal showed nonoverlapping single centromeric (red) and telomeric (green) signals in one allele of tumor cells, whereas the other allele (not rearranged) showed yellow signals due to superimposed red and green signals. Tumors with greater than or equal to $10 \%$ abnormal cells were considered positive for rearrangement. ${ }^{4}$

\section{Discussion}

Tracheal ACC is a rare low-grade malignant tumor of the trachea and bronchi. It is most common in the lower third of the main bronchus and can occur at any age with a peak 


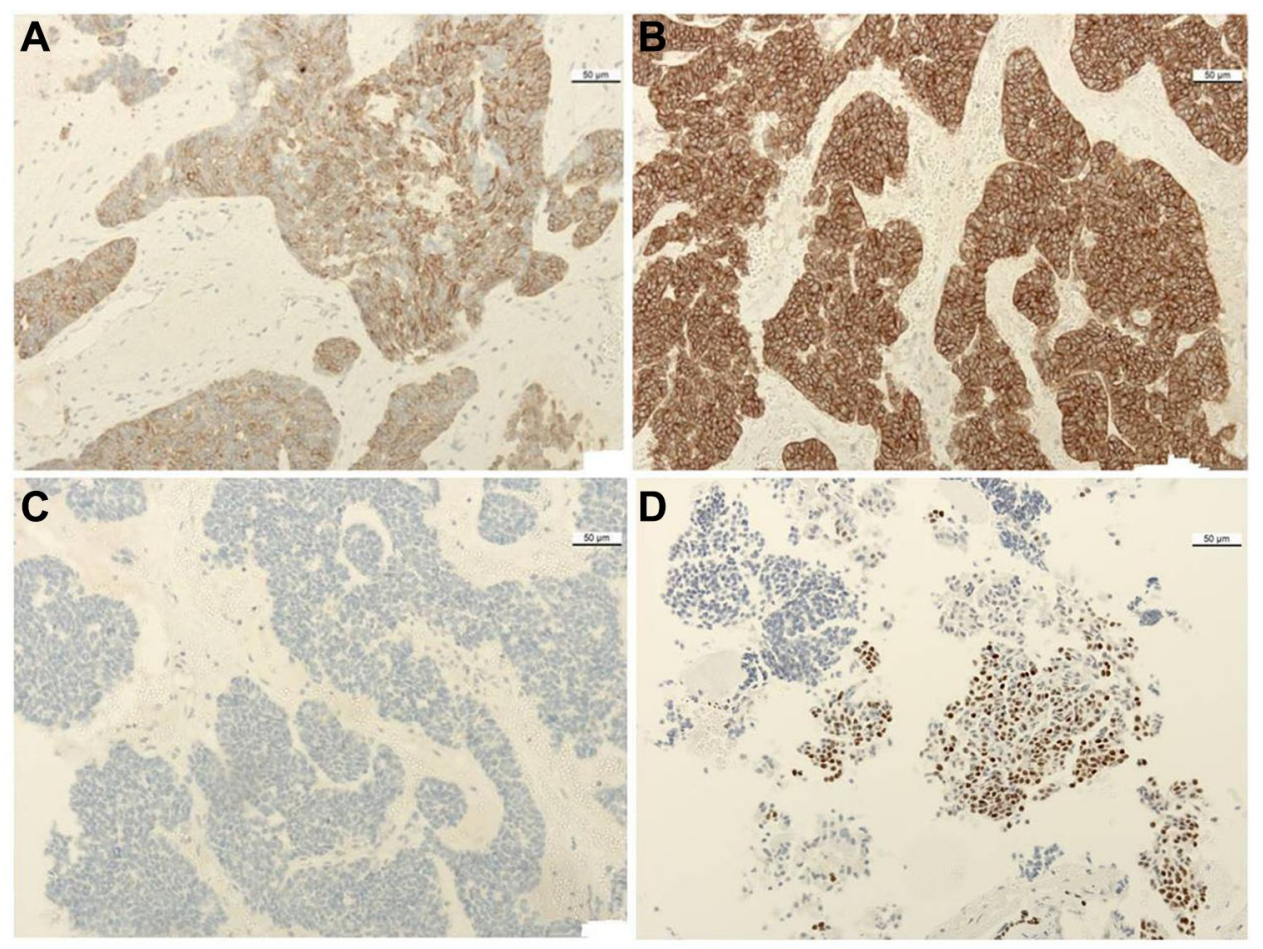

Figure 4 Immunohistochemical analysis of the tumor. (A) CK7 was positive in some cells of solid cell nests. (B) The expression of CDI I7 was diffusely positive in tumor cells. (C) p63 was negative in the majority of the neoplastic cells. (D) Focal staining was seen in a small area for p63.

incidence of $40-50$ years. $^{5}$ For primary tracheal tumors, although the fiberoptic bronchoscopy detection rate is low, it is unique for early occult lesions and preoperative pathological diagnosis. In our case, fiberbronchoscopy revealed new organism on the left and front walls of the lower part of the trachea, extending from $3 \mathrm{~cm}$ above the protrusion to the upper left main bronchus. The clinical features of this patient and the presence of bronchoscopy are very similar to those reported in the literature.

Table I Immunohistochemical Features of the Tumor

\begin{tabular}{|l|l|}
\hline Antibody & \multicolumn{1}{|c|}{ IHC Pattern } \\
\hline $\begin{array}{l}\text { CK, CDII7 } \\
\text { CK7, CK5/6 }\end{array}$ & $\begin{array}{l}+ \text {, diffuse } \\
+, \text { local }\end{array}$ \\
\hline p63 & - , (focal weak staining) \\
\hline Vimentin, CD99 & + , local \\
\hline TTF-I, NapsinA & - \\
\hline P40 & - \\
\hline LCA & - \\
\hline Syn, ChgA, CD56, NSE & - \\
\hline FLI-I, TDT & - \\
\hline
\end{tabular}

Primary tracheal ACC is a rare, distinctive salivary gland-type malignant neoplasm. The tumor is composed of a dual-cell population of luminal and myoepithelial or basal cells. ACC has three distinct growth patterns: tubular, cribriform, and solid. The solid variant appears to be a high-grade subtype when compared with classical ACC. It is more aggressive, prone to distant metastasis, and has a worse prognosis. ${ }^{2,3}$ The tumor cells in the solid variant

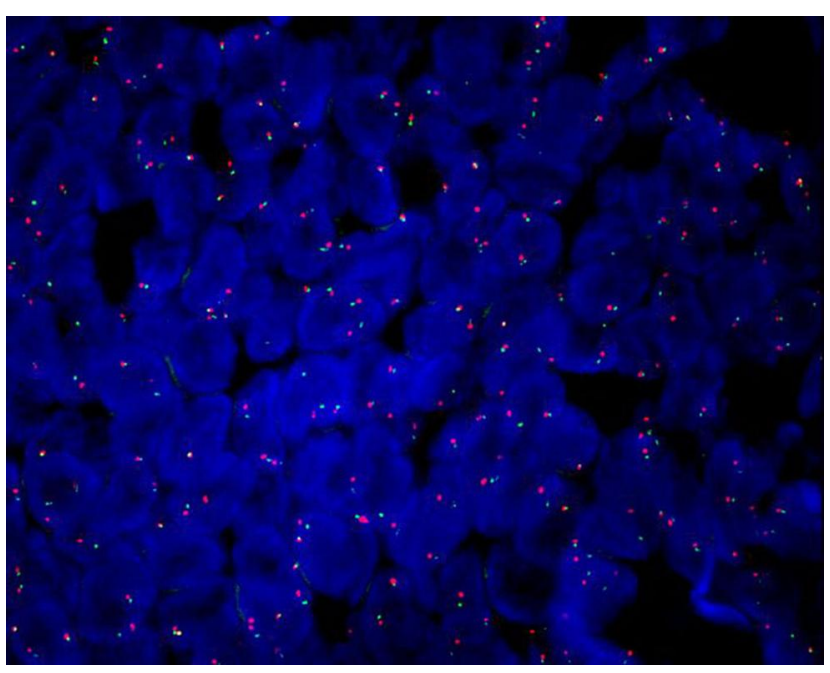

Figure 5 FISH for MYB break-apart assay showed separate red and green signals in more than $30 \%$ of tumor cells. 
exhibited moderate to marked atypia and pleomorphism. Increased mitotic activity is also commonly seen. Some of the cells surrounding the solid nests were arranged in a palisade. In our case, in small areas that constituted less than $5 \%$ of the neoplasm, inconspicuous glandular lumen or cribriform structure can also be seen. Although very focal, the presence of cribriform structures is the key morphological feature for diagnosis. ${ }^{6,7}$ The local cribriform structure can often reveal the nature of adenoid cystic carcinoma.

The two different cell types of ACC can be easily distinguished by immunohistochemistry. The basal layer cells are typically positive for myoepithelial markers (p63, S-100, Calponin), and vimentin, whereas the luminal cells exhibit positivity for CK7, EMA, CK5/6, and c-kit (CD117). In our case, the tumor was uniformly positive for CK and CD117, with focal expression of CK7 and CK5/6, while myoepithelial cells were only rarely demonstrable with p63. Previous studies have revealed that the typical dual population of cell types is not always demonstrable in the solid variant of ACC. ${ }^{7}$ This is in concordance with our histologic results. The tumor is entirely composed of basaloid cells with only rare foci of myoepithelial cells. It has been suggested that basaloid cells represent primitive cells. They may be poorly differentiated or undifferentiated tumor cells with the potential of multi-directional differentiation into adenoid, squamous, and myoepithelial cells. In solid variants of ACC with distinctive basaloid features, solid nests may be scattered with inconspicuous adenoid or sieve structures, which suggest an undifferentiated or poorly differentiated ACC. Consequently, the absence of cells expressing myoepithelial markers does not exclude the diagnosis of solid variant ACC. ${ }^{6,7}$

Person et al confirmed the presence of translocation: $\mathrm{t}(6 ; 9)(\mathrm{q} 22-23 ; \mathrm{p} 23-24)$ in ACC, which led to the formation of an MYB-NFIB fusion gene and has been identified as a tumor-specific cytogenetic abnormality of ACC. The consequence of the translocation leads to overexpression of MYB-NFIB transcripts and activation of MYB target genes involved with cell cycle control, apoptosis, cell adhesion, and angiogenesis. ${ }^{6,8}$ The incidence of MYB-NFIB fusion in ACC was $49-57 \%$ (mean $54 \%$ ), and it is highly specific. ${ }^{8}$ Detection of MYB rearrangement can be used as a reliable and accurate method for differential diagnosis between ACC and other tumors. In our case, FISH for MYB break-apart assay showed separate red and green signals in more than $30 \%$ of tumor cells, supporting the diagnosis of solid variants of ACC. However, it should be emphasized that not all ACCs show an MYB rearrangement, and a normal FISH pattern does not rule out this diagnosis. Xie et al found that the coexpression of Notch1 and FABP7 is strongly associated with poor survival in resected tracheobronchial ACC. ${ }^{9}$ Pourabdollah Toutkaboni et al collected 26 trachea/bronchus ACC patients and found that the marker mTOR was positive in $46 \%$ of the cases. ${ }^{10}$

The differential diagnosis includes several other small round tumors. ${ }^{6,11,12}$ Poorly differentiated squamous cell carcinoma is composed of nests or cords of cells and have pseudoglandular structures without obvious keratinization. Frequently, in situ carcinoma of squamous cells is found near the infiltrating area. P63 and CK5/6 are typically diffusely and strongly positive, whereas CD117 is negative. Small-cell (neuroendocrine) carcinoma shows similar features of tightly packed rounded or ovoid cells with hyperchromatic nuclei, scanty cytoplasm, and nuclear molding. Markers of neural differentiation are variably expressed. CK may show a paranuclear, dot-like expression pattern. Nuclear TTF-1 expression may be prominent in more than $90 \%$ of cases, with different degrees of bcl-2 and CD117 positivity. The ki-67 positive rate is usually more than $50 \%$. Small-cell carcinoma is generally free of the typical cribriform structure in adenoid cystic carcinoma. Nonkeratinizing squamous cell carcinoma (SCC) with basaloid features is a highly aggressive tumor with a worse prognosis. It mainly consists of clusters of basallike cells, which are palisade-arranged around the tumor. CK5/6 and p63 have been shown to be positive in 90-100\% of basaloid SCC. Ewing's sarcoma (ES) and primitive neuroectodermal tumor (PNET) cells are usually very bland and monotonous, sometimes with small amounts of clear cytoplasm, and are diffusely membranously positive for CD99. The tumor frequently shows rearrangements of the EWSR1 gene, most commonly leading to fusions with the FLI1 gene. Mucosal melanoma is typically diffusely positive for S- 100 protein and melanocytic markers. The tumor cells can be arranged in solid, glandular, epithelioma, and sarcomatoid form. In addition, tracheal ACC needs to be differentiated from other primary tracheal malignancies, especially tracheal salivary gland-type tumors, such as basal cell adenocarcinoma and epithelial-myoepithelial carcinoma.

Because of its growth characteristics, tracheal ACC is prone to local recurrence and distant metastasis. The surgical resection approach depends on the location of the 
tumor in the airway. Surgical resection is the optimal management in most cases. ${ }^{13}$ However, in several studies, surgical resection followed by postoperative adjuvant radiotherapy had a better prognosis and hence is a preferred mode of treatment. ${ }^{14}$

\section{Conclusion}

In summary, we report on a case of a solid variant of tracheal ACC with basaloid features. Due to its relatively complex tissue structure and certain limitations in bronchoscopy biopsy, it is concluded that in the case of solid aggregates predominated by poorly differentiated basaloid cells, primary tracheal ACC should be considered as an important differential diagnosis. Additional immunohistochemistry and molecular techniques could be applied to refine the diagnosis. This case report highlights the significance of various histological patterns and diagnostic modalities in making an accurate diagnosis of primary tracheal ACC at an early stage.

\section{Abbreviations}

ACC, adenoid cystic carcinoma; CT, computed tomography; PET, positron emission tomography; FNA, fine needle aspiration; FISH, Fluorescence in situ hybridization.

\section{Ethics Approval and Consent to Participate}

This study was conducted with approval from the Ethics Committee of Shanghai General Hospital, Shanghai Jiao Tong University School of Medicine. This study was conducted in accordance with the declaration of Helsinki. Written informed consent was obtained from the patient for the publication of this case report and accompanying images.

\section{Disclosure}

The authors declare that they have no competing interests.

\section{References}

1. Moran CA. Primary salivary gland-type tumors of the lung. Semin Diagn Pathol. 1995;12:106-122.
2. Thompson LD, Penner C, Ho NJ, et al. Sinonasal tract and nasopharyngeal adenoid cystic carcinoma: a clinicopathologic and immunophenotypic study of 86 cases. Head Neck Pathol. 2014;8:88-109. doi:10.1007/s12105-013-0487-3

3. van Weert S, van der Waal I, Witte BI, Leemans CR, Bloemena E. Histopathological grading of adenoid cystic carcinoma of the head and neck: analysis of currently used grading systems and proposal for a simplified grading scheme. Oral Oncol. 2015;51:71-76. doi:10.1016/j.oraloncology.2014.10.007

4. Wetterskog D, Lopez-Garcia MA, Lambros MB, et al. Adenoid cystic carcinomas constitute a genomically distinct subgroup of triple-negative and basal-like breast cancers. $J$ Pathol. 2012;226:84-96. doi:10.1002/path.2974

5. Zhao Y, Zhao H, Fan L, et al. Adenoid cystic carcinoma in the bronchus behaves more aggressively than its tracheal counterpart. Ann Thorac Surg. 2013;96:1998-2004. doi:10.1016/j.athoracsur.2013.08.009

6. Ben Salha I, Bhide S, Mourtzoukou D, Fisher C, Thway K. Solid variant of adenoid cystic carcinoma: difficulties in diagnostic recognition. Int J Surg Pathol. 2016;24:419-424. doi:10.1177/ 1066896916642011

7. Shin SJ, Rosen PP. Solid variant of mammary adenoid cystic carcinoma with basaloid features: a study of nine cases. Am J Surg Pathol. 2002;26:413-420. doi:10.1097/00000478-200204000-00002

8. Persson M, Andren Y, Mark J, et al. Recurrent fusion of MYB and NFIB transcription factor genes in carcinomas of the breast and head and neck. Proc Natl Acad Sci U S A. 2009;106:18740-18744. doi:10.1073/pnas.0909114106

9. Xie M, Wu X, Zhang J, et al. The prognostic significance of Notch1 and fatty acid binding protein 7 (FABP7) expression in resected tracheobronchial adenoid cystic carcinoma: a multicenter retrospective study. Cancer Res Treat. 2018;50:1064-1073. doi:10.4143/ crt.2017.337

10. Pourabdollah toutkaboni M, Farahani M, Sadegh A, et al. The incidence of mTOR marker in tracheal adenoid cystic carcinoma by immunohistochemical staining. Adv Respir Med. 2020;88:305-312. doi:10.5603/ARM.a2020.0120

11. Emanuel P, Wang B, Wu M, Burstein DE. p63 Immunohistochemistry in the distinction of adenoid cystic carcinoma from basaloid squamous cell carcinoma. Mod Pathol. 2005;18:645-650. doi:10.1038/modpathol.3800329

12. Cabibi D, Cipolla C, Maria florena A, et al. Solid variant of mammary "adenoid cystic carcinoma with basaloid features" merging with "small cell carcinoma". Pathol Res Pract. 2005;201:705-711. doi:10.1016/j.prp.2005.04.012

13. Scherl S, Alon EE, Karle WE, et al. Rare tracheal tumours and lesions initially diagnosed as isolated differentiated thyroid cancers. Thyroid. 2013;23:79-83. doi:10.1089/thy.2012.0192

14. Calzada AP, Miller M, Lai CK, Elashoff DA, Abemayor E, St John MA. Adenoid cystic carcinoma of the airway: a 30-year review at one institution. Am J Otolaryngol. 2012;33:226-231. doi:10.1016/ j.amjoto.2011.07.003
OncoTargets and Therapy

\section{Publish your work in this journal}

OncoTargets and Therapy is an international, peer-reviewed, open access journal focusing on the pathological basis of all cancers, potential targets for therapy and treatment protocols employed to improve the management of cancer patients. The journal also focuses on the impact of management programs and new therapeutic

\section{Dovepress}

agents and protocols on patient perspectives such as quality of life, adherence and satisfaction. The manuscript management system is completely online and includes a very quick and fair peer-review system, which is all easy to use. Visit http://www.dovepress.com/ testimonials.php to read real quotes from published authors. 